\title{
Severe obstructive pattern mimicking an asthma exacerbation as first presentation of crack smoking: A case report
}

\author{
Thomas Galasso, Andrea Mazzetta, Valentina Conio, Elena Paracchini, Sara Surbone, Eti Maria \\ Giulia Di Vincenzo, I sa Cerveri, Federica Meloni \\ Department of Molecular Medicine, University of Pavia, IRCCS San Matteo, Pavia, Italy.
}

Correspondence: Thomas Galasso. Address: Department of Molecular Medicine, University of Pavia, IRCCS San Matteo, Pavia, Italy. Email: tommk84@yahoo.it

Received: December 5, 2014

Accepted: February 26, $2015 \quad$ Online Published: March 27, 2015

DOI : $10.5430 /$ crim.v2n2p60

URL: http://dx.doi.org/10.5430/crim.v2n2p60

\begin{abstract}
Crack inhalation is known to cause a wide spectrum of acute and chronic pulmonary complications, along with imaging manifestations and lung function patterns. The diagnosis of these conditions is often delayed or mistaken. Only little information about the management and follow up of these pulmonary complications is available in literature. We present the case of a young patient referred to our clinic for symptoms mimicking an asthma exacerbation, not responsive to inhalant treatment. After investigations and the admission of crack abuse by the patient a final diagnosis of bronchiolitis crack-related was made. The patient was then followed for more than a year showing that lung function tests may be useful both for the diagnosis and follow up of such pulmonary complications.
\end{abstract}

\section{Keywords}

Crack abuse, Obstructive pattern, Follow up

\section{I ntroduction}

Cocaine is one of the most widely used illicit drugs. It is usually taken intra nasally in the hydrochloride form; there are also two forms, obtained with chemical process, by which cocaine can be smoked: freebase and crack cocaine.

Respiratory symptoms are common after crack inhalation; they include dry or productive cough, wheezing, dyspnoea, chest pain and haemoptysis ${ }^{[1,2]}$. There is a large spectrum of imaging manifestations, mostly reported in literature as case reports, including ground glass opacities, consolidations, peribronchial nodules, inter and intralobular interstitium thickening, honeycombing ${ }^{[3]}$. Symptoms and imaging manifestation can be expression of a wide range of acute or chronic pulmonary complications: pulmonary oedema, diffuse pulmonary alveolar haemorrage, acute asthma exacerbations, acute and chronic eosinophilic lung infiltrates (the former known as "crack lung” syndrome), nonspecific interstitial pneumonia and bronchiolitis obliterans with organizing pneumonia.

The diagnosis is often delayed or mistaken because of the different presentations of respiratory damage and the fact that abuse is not usually declared by patients. The role of lung function test has not been yet pointed out both for the diagnosis 
and monitoring. Only little information about the management and follow up of these pulmonary complications is available in literature.

\section{Case presentation}

In November 2012, a 19-year-old woman, mild cigarette-smoker without previous history of asthma, allergy or any respiratory problem, was referred to our outpatient clinic for evaluation of dyspnoea on exertion, chest tightness and productive cough persisting over the previous 6 months. Within this period she was treated by general practitioner with several antibiotic cycles and inhaler therapy (inhaled corticosteroid + long acting beta-agonists). In occasion of onset of fever and exacerbations of respiratory symptoms chest x-ray was performed, showing focal opacity in the upper right lobe, then treated as a community acquired pneumonia. At our visit we notice that her BMI was under lower limit of normal $\left(15 \mathrm{~kg} / \mathrm{m}^{2}\right)$. Physical examination revealed diffuse wheezing and rales. Arterial blood gas analysis revealed $\mathrm{PaO}_{2} 72.5$ mmHg, $\mathrm{PaCO}_{2} 32 \mathrm{mmHg}, \mathrm{pH} 7.4$, $\mathrm{SatO}_{2} 96 \%$. Chest x-ray was normal. In suspicion of an acute exacerbation of asthma she was discharged with a prescription of oral corticosteroids and inhaler therapy (CSI+LABA). On follow up after 3 weeks, the patient reported an initial clinical response, but few days later, after sudden withdrawal of steroid, her symptoms flared up. Thus, she was hospitalized for further evaluation. A chest X-ray showed mild diffuse interstitial thickening without focal opacities. Blood tests were performed with evidence of mild eosinophilia $(10.8 \%, 800 / \mathrm{mcl})$. A flow-volume curve, determination of lung volumes (pre and post bronchodilator with salbutamol $400 \mathrm{mcg}$ ) and diffusing capacity test were performed, showing a severe obstructive pattern (FEV1 1.70 L 46\% pred, FVC 2.50 L 60\% pred, FEV1/FVC 67\%), severe hyperinflation (RV 2.64 L, 182\% pred) and decreased carbon dioxide diffusing capacity (DLCO $37 \%$ pred). After administration of salbutamol $400 \mathrm{mcg}$ there was a significant bronchodilatation and a huge improvement of the hyperinflation (see Table 1). Unfortunately, after 2 days of hospitalization, the patient became extremely nervous and irritable and, against our opinion, she decided to leave. In this occasion we became aware of the difficult relationships between the patient and her parents; but it was impossible to examine the familiar dynamics in depth.

Table 1. Comparison between respiratory function tests at first evaluation (before and after administration of salbutamol $400 \mathrm{mcg})$

\begin{tabular}{lllll}
\hline & Pre-bronchodilator & & \multicolumn{2}{l}{ Post-bronchodilator } \\
\hline & Absolute value & \% of predicted & Absolute value & \%, change of baseline \\
FEV1, $L$ & 1,70 & 46 & 1,96 & +7 \\
FVC, $L$ & 2,52 & 61 & 2,67 & +4 \\
VC, $L$ & 2,52 & 60 & 2,67 & +4 \\
FEV1/VC & 67 & 79 & 73 & +7 \\
TLC, $L$ & 5,16 & 93 & 4,50 & -12 \\
RV, $L$ & 2,64 & 182 & 1,83 & -55 \\
DLCO & 3,80 & 37 & & \\
\hline
\end{tabular}

In December 2012 she performed a chest HRCT as outpatient, which showed bilateral mild ground glass opacities with lobular distribution, some focal flogistic opacities, diffuse bronchial and bronchiolar wall thickening, and presence of mosaic perfusion and air trapping (see Figure 1A and 1B).

Finally, after an initial denial of a new hospitalization, a week later the patient accepted to admit herself to hospital. There, she was submitted to bronchoscopy and additional tests. At bronchoscopy there was diffuse evidence of a hyperaemic and flogistic bronchial mucosa with secretions. BAL was performed, which showed an increased total cellularity with $25 \%$ of lymphocytic cells and $11 \%$ of eosinophilic cells, CD4/CD8 ratio below the normal range (0.22). Microbiological tests were negative for aerobic bacteria, micobacteria and fungi. The transbronchial biopsy (TBB) performed in the anterior branch of upper right lobe demonstrated inflammatory aggregate with large amount of pigmented macrophages and 
eosinophilic granulocytes. Blood tests showed mild autoantibody positivity (ANA positivity, 1:160); other test were normal (including ACE-s, lysozime, Ig dosage, Ig E anti Aspergillus fumigatus and anti Candida albicans, thyroid hormones). Meanwhile, the pathological BMI with a weight loss of $10 \mathrm{~kg}$ in the last year, her very young age and a behaviour characterized by fits of rage misdirected us toward a suspicion of anorexia. Thus we asked for a psychiatric evaluation but, in spite our advice, she decided to immediately go home without attending the visit.

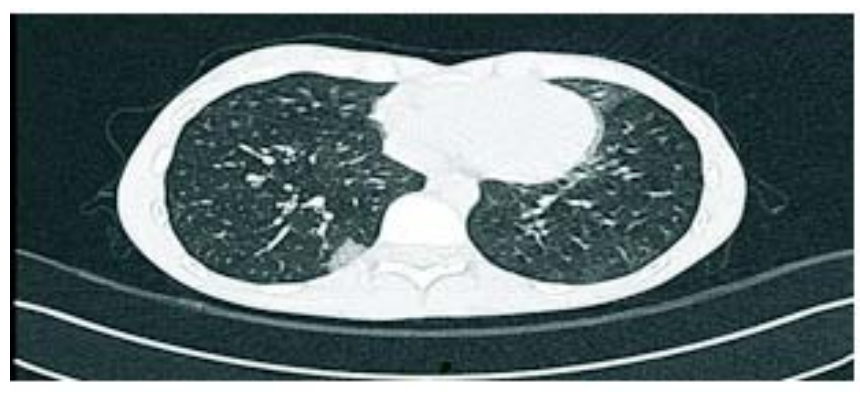

Figure 1A. Focal ground glass opacities in first CT scan
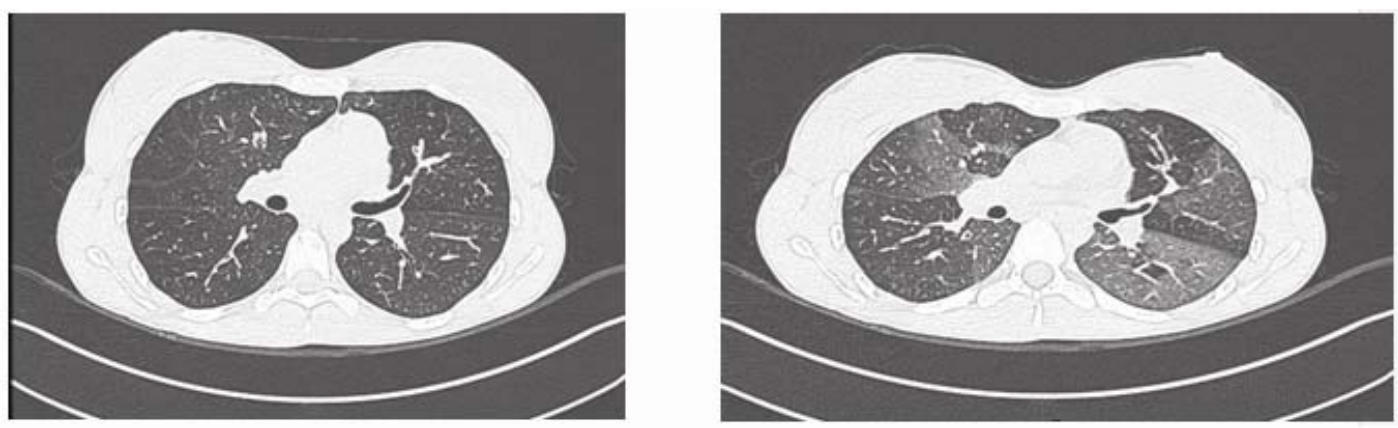

Figure 1B. Mosaic perfusion due to air trapping in the expiratory phase in first CT scan

A few days later, the patient was admitted to the local emergency room for multiple traumatic contusions and severe restlessness. Her father referred an episode of self-harming and aggression towards others, caused by drugs abuse. Toxic tests were positive for cocaine, cannabis and opioids and the patient was admitted. According to her father, the patient only recently admitted smoking of crack-cocaine, marijuana and heroin, lasting more than a year. The psychiatric physician decided for admission in the psychiatric service in order to start rehab. At this point a clinical diagnosis suggestive for bronchiolitis secondary to crack smoking was made. Prednisone $37.5 \mathrm{mg}$ daily and inhaler therapy (CSI+LABA) was prescribed. A few days later she moved to a psychiatric rehab where she was treated with neuroleptic drugs and supportive psychotherapy. At discharge she refused the proposed rehabilitation center and/or individual psychotherapy but accepted to undergo urinary toxicological monitoring and close pulmonary follow up. After 2 months the patient was asyntomatic, her toxicological test was negative and the PFT demonstrated an excellent improvement with lung volumes at the lower limit of normality (FEV1 3.31 L 90\% pred, FVC 3.42 L 82\% pred, FEV1/FVC 96\%) but only a moderate improvement in DLCO (58\% pred). At the chest HRCT there was no more evidence of ground glass opacity or mosaic attenuation (see Figure 2).

Unfortunately, along the year, we observed a fluctuation in the lung function values particularly in FEV1, partially due to the tapering of oral corticosteroids and partially to the reprise of crack smoking as documented by positivity at toxicological tests; the latter data were not immediately available at the time of each visit due to the low compliance of the patient (see Figure 3). In two occasions in which the PFT were particularly worsened we performed chest HRCT scans which showed new migrant ground glass opacities, bronchiolar filling and expiratory air trapping. 


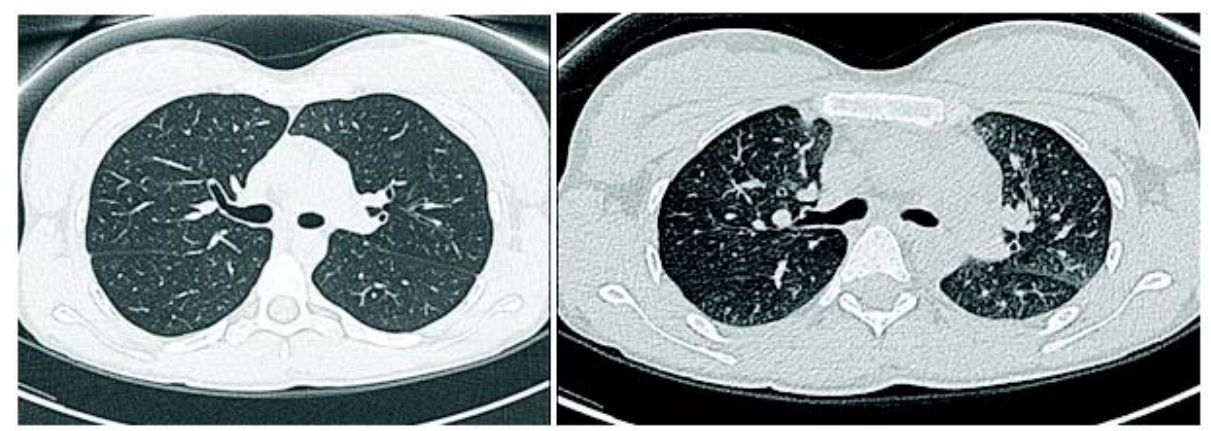

Figure 2. Resolution of ground glass opacities and mosaic perfusion in the expiratory phase

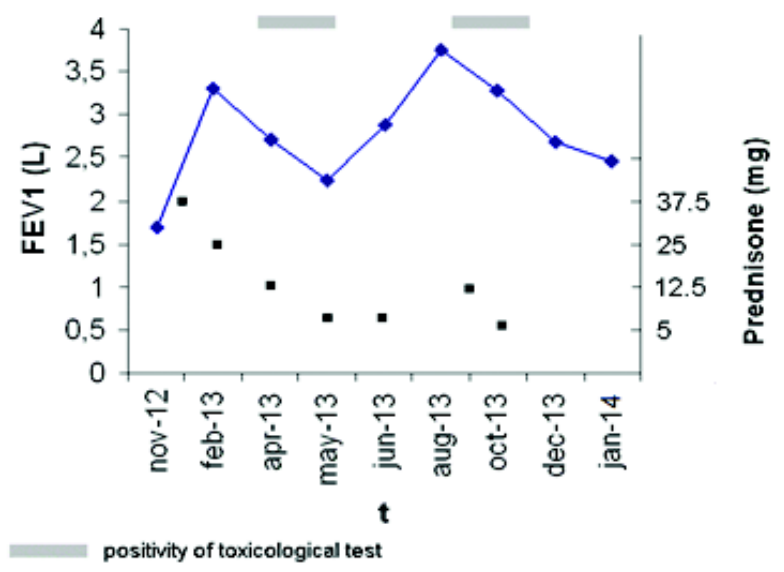

Figure 3. Trend of FEV1 values with concomitant dose of steroid therapy and positivity at toxicological test during the follow up period

\section{Discussion}

Illicit inhaled drug abuse has become more common, increasing concern about potential airway complications.

Cocaine hydrochloride is an alkaloid extracted from the Erythroxylon coca plant after a chemical process ${ }^{[4]}$. Freebase form is obtained dissolving cocaine hydrochloride in water, then adding a base (eg, ammonia) and a solvent (ether or alcohol) ${ }^{[5]}$. Crack cocaine is obtained by dissolving cocaine hydrochloride in water with sodium bicarbonate (baking soda) to extract the hydrochloride and make the substrate heat stable. The cocaine base precipitates forming hard masses or rocks that melt when dry, but vaporize at high temperatures, producing crackles when heat.

It has been demonstrated that the average size of the particles generated in the combustion of crack cocaine is small enough to result in their deposition in the alveolar spaces ${ }^{[6]}$ and that there is an extensive accumulation of large amounts of macrophagic and extracellular carbon pigment ${ }^{[7]}$ and damage of the alveolar-capillary membrane.

Respiratory symptoms as cough, wheezing, dyspnoea, chest pain and haemoptysis are quite common after crack inhalation. The diagnosis is frequently delayed or inappropriate due to a broad range of histopathological changes and difficulty to achieve personal information about life style and social attitude. Some reports described clinical cases misdiagnosed with asthma, Churg Strauss syndrome or sarcoidosis ${ }^{[8-10]}$.

In our experience CT scan showed the presence of mosaic perfusion and air trapping, a sign related to bronchiolitis but not asthma exacerbation $^{[11]}$. The delay in detecting the causative role of crack-smoking was due to the misunderstanding of 
the clinical presentation, since the age, lower BMI and behaviour of the patient were more suggestive for anorexia than drug-addiction but also to the impossibility of performing further evaluations because of the early self-discharge in two occasions and denial of a new hospitalization. The final diagnosis of bronchiolitis due to crack abuse was set only after the admission to the local emergency room for traumatic contusions and psychomotor agitation and the recent discovery of the drug abuse by her father.

At our knowledge there are no case reports documenting prolonged follow up of patients during crack abuse. In our case a good correlation between pulmonary function tests and CT features was found in the monitoring of the disease, suggesting that PFTs could be a reliable substitute, with obvious benefit for the patient. The systemic corticosteroid therapy associated with inhaler therapy and the complete weaning from abuse for 2 months led to a normalization of the pulmonary features. The subsequent worsening in symptoms, lung function and imaging, seen at least in 2 occasions, was correlated with positive toxicological test but also in part with the tapering of the oral steroids. Taking into account the age of the patient and collateral effects of systemic steroids, we gave high priority to the weaning from crack abuse instead of increasing the steroid dose.

The patient accepted the advice given by the psychiatric team and strongly supported by us to start rehab.

\section{Conclusion}

In conclusion, we report here a case of bronchiolitis crack-related with a year-follow up. The presentation as an asthma exacerbation not responsive to treatment must arise the suspicion of bronchiolitis and further investigation should be planned. Moreover this report highlights the importance of high index of suspicion regarding drug abuse during the diagnostic work out of respiratory symptoms.

Clinicians should be reminded that it is important to attain confidential information about lifestyle and social attitudes, so that an appropriate and early diagnosis can be made. In case of a suspected addictive drug related-pulmonary disease a tight collaboration between pulmonologist and psychiatric physician is highly needed during the diagnosing and monitoring process.

\section{References}

[1] Carlos S. Restrepo, Jorge A. Carrillo, Santiago Martínez, et al. Pulmonary complications from Cocaine and Cocaine-based substances: Imaging manifestations. Radiographics. 2007; 27: 4.

[2] Alexandre Mançano, Edson Marchiori, Gláucia Zanetti, et al. Pulmonary complications of crack cocaine use: high-resolution computed tomography of the chest. J Bras Pneumol. 2008; 34(5): 323-7

[3] Michael B. Gotway, Shelley R. Marder, Douglas K. Hanks, et al. Thoracic complications of illicit drug use: an organ system approach. Radiographics. 2002; 22 Spec: S119-35.

[4] Casale J, Klein R. Illicit production of cocaine. Forensic SciRev S. 1993; 95-107.

[5] Warner EA. Cocaine abuse. Ann Intern Med. 1993; 119: 226-35. PMid:8323092 http://dx.doi.org/10.7326/0003-4819-119-3-199308010-00009

[6] Carroll A. Snyder, Ronald W. Wood, John F. Graefe, et al. "Crack smoke” is a respirable aerosol of cocaine base. Pharmacol Biochem Behav. 1988; 29: 93-5.

[7] Greenebaum E, Copeland A, Grewal R, et al. Blackened bronchoalveolar lavage fluid in crack smokers: a preliminary study. Am J Clin Pathol. 1993; 100: 481-7.

[8] Fiorella Calabrese, Domenico Barrile, Giampietro Frison, et al. An asthmatic patient with progressive lung dysfunction: a case of misdiagnosis. Lancet. 2006; 368: 814.

[9] R Orriols, X Munoz, J Ferrer, et al. Cocaine-induced Churg Strauss vasculitis. Eur Respir J. 1996 Jan; 9(1): 175-7.

[10] Bisconti, et al. Crack cocaine snort induced granulomatosis and pulmonary fibrosis mimicking sarcoidosis. Rassegna Pat App Resp. 2013; 28: $47-49$

[11] S.P Jensen, D.A Lynch, K.K Brown, et al. High-resolution CT features of severe asthma and bronchiolitis obliterans. Clinical Radiology. 2002; 57: 1078-85. 